\title{
MODELING AND MANAGING FEDERATED WEB-BASED SYSTEMS
}

\author{
Johannes Meinecke ${ }^{1}$, Martin Gaedke ${ }^{2}$, Frederic Majer ${ }^{1}$, Alexander Brändle ${ }^{3}$ \\ ${ }^{1}$ Institute of Telematics, University of Karlsruhe, Engesserstr. 4, 76128 Karlsruhe, Germany \\ meinecke@tm.uka.de,majer@tm.uka.de \\ ${ }^{2}$ Distributed and Self-organizing Computer Systems Group, Chemnitz University of Technology Straße der Nationen 62, \\ 09107 Chemnitz, Germany \\ gaedke@informatik.tu-chemnitz.de \\ ${ }^{3}$ Microsoft Research Cambridge, 7 JJ Thomson Avenue, CB3 OFB Cambridge, United Kingdom \\ alexbr@microsoft.com
}

Keywords: $\quad$ Security, Web Services, Architecture, Modeling, Federation, Service Architectures

\begin{abstract}
Among the various aspects of Web applications that are subject to modeling, like navigation, interaction or business processes, the architectural aspect is receiving growing attention. This is related to the fact that the Web is increasingly used as a platform for distributed services which transcend organizational boundaries to form so-called federated applications. In this context, we use the term "architecture" to denote the composition of the overall solution into individual Web applications and Web services that belong to different parties and invoke each other. The design and evolution of such systems calls for models that give an overview of the federation structure and reflect the technical details of the various accesses. We introduce the WebComposition Architecture Model (WAM) as an overall modeling approach tailored to aspects of highly distributed systems with federation as an integral factor.
\end{abstract}

\section{INTRODUCTION}

The needs of modern businesses that operate worldwide, cooperate with various partners, and deliver their services in real-time pose complex tasks to be solved by technological disciplines. Over the years, this trend of connected businesses has affected the Web and lead to a change towards a platform for distributed applications. Beyond merely supplying documents to users, it has recently been used as a communication infrastructure that links together applications, e.g. by exposing functionality through Web services. Now, a tendency can be observed towards a new class of applications that is made possible by these technological advancements: the federated portal respectively $4^{\text {th }}$ generation portal (Gootzit and Phifer 2003). The relationships within such federations of portals belonging to multiple organizations do not only consist of simple HTML links, but comprise the connection of the portal backbones, i.e. they also share for example data, functionality or user accounts.

However, the added business value brought by the new sophisticated approaches does not come for free. Besides the challenges involved with federating applications on a semantic level, e.g. addressed in (Park and Ram 2004), the typical characteristics of these enabled solutions cause a high degree of technical complexity. In the context of federation, the question of access control and security is especially important and requires the application of advanced concepts (Cameron 2005). This is related to the fact that in such scenarios, external users from cooperating organizations have to be granted access to local resources while preserving the autonomy of the individual federation partners. All system characteristics are subject to change during the evolution of the federation and its components. During the system lifetime, new services may be added, removed or substituted by others and new partners can join or leave the federation.

The mentioned factors accumulate to a complexity that requires systematic methods for modeling, building and operating concrete systems. To this end, we propose the WebComposition Architecture Model (WAM), which we describe in the remainder of the paper. We identify three key requirements that we believe to be vital for modeling 
the architecture of the outlined type of application. We then provide a broad overview of related modeling languages and introduce our own WAM approach as well as an XML-based notation that can be used to provide WAM models in a machinereadable way. This format is used by a service-based support system, whose application within an integration project is outlined.

\section{MODELLING CHALLENGES}

When the architecture of a system is modeled, there is a wide range of different potential aspects to be described and viewpoints to be taken. From a software engineering point of view, (Bass, Clements et al. 1998) defines the term architecture as "the structure or structures of the system, which comprise software components, the externally visible properties of those components, and the relationships among them". In our federation- and Web-specific case, the system can be conceived as the federation that consists of Web services and applications forming the components. To establish some guidance for a modeling approach targeted at such architectures, we have derived the following key requirements from the system characteristics.

\subsection{Challenges}

Integration of security concerns into architecture models: One of the described particularities of the targeted type of application is the fact that accesses transcend organizational boundaries. In this context, the term security is related to the precautions to secure authentication and authorization, which is necessary to guarantee compliance with the partners' access policies. In conventional solutions with only one organization involved, this can be realized with a zone concept. This is often achieved by combining firewalls and virtual private networks (VPN) with implicit trust between the system parts inside the zone. Here, the focus often lies on modeling the primary functional structure first and taking security into account later on. The major difference in federated applications is the existence of not just one zone, but multiple zones; with the accesses depending on trust relationships between the controlling organizations. This underlying trust network may also affect the structure of the system, e.g. with respect to whether a service can be used by an application or not. Consequently, we propose the integration of security considerations into the same model that is already being used to describe the system architecture.

Hiding unwanted complexity: Models of federated systems have to cope with a high degree of complexity, partially caused by advanced federationenabled security protocols, as e.g. token-based approaches. For example, when a user logs into a portal and queries information from a service, this may involve the exchange of a high number of messages in order for the token to be requested, issued and transported to the protected service before the access is granted. While this could be modeled e.g. with a UML sequence diagram, the exact succession of calls may in many cases have no relevance for the real modeling purpose. What is rather required is an abstraction from such details that actually supports the process of communicating the model to others. Preferably, we are interested in a notation that is simple enough to be drawn using pen and paper to sketch the most important facts and powerful enough to add further details on demand.

Bridging the gap between model and system: The fact that federations are subject to frequent changes can cause a gap between the model and the modeled reality. If the model takes the form of a diagram on paper or in a document with a vendor-specific format, it is in danger of being constantly outdated and therefore useless to work with. In order to close this gap quickly enough, one approach is to rely on machine-readable representations that can be processed by tools. Hence, this would enable automatic updates of the model (system to model), as well as make the contained metadata available to operations that change, maintain or otherwise support the system (model to system).

\subsection{Related work}

In the following, several existing approaches facing the challenge of modeling the architecture of highly distributed and interconnected Web-based systems will be described briefly and examined regarding our derived key requirements.

The first generation of architecture modeling approaches was focusing on a separation of concerns by specifying dedicated layers for different aspects of the system. These approaches share a common view on the importance of some concerns. Kirchner (Kirchner 2005) specifies a Business Process Layer, a Software Layer and a Hardware Layer. The project ARCUS (Hermanns, Jänsch et al. 1999), which aimed at architectural support for systems in the banking sector, introduced an additional layer for the 
specification of technical terms regarding the problem domain. Still, both approaches are designed to reflect the system architecture of an enterprise and do not take identities and applications transcending organizational boundaries into account. Support through machine-readable system descriptions is also beyond their scope.

More recently, new approaches like the Dynamic Systems Initiative (DSI), the Data Center Markup Language (DCML) and the Systems Modeling Language (SysML) have been introduced that try to close the gap between model and system. DSI is a technological strategy devised by Microsoft that aims at an integrative support for the design, deployment and operation of distributed systems (Microsoft 2003). The initiative is driven by the idea of combining the two processes of building and operating IT solutions to emphasize the application life cycle as a whole. Although the approach deals with modeling systems in general, its major focus is on the Windows platform. DCML is an approach to describe data center environments, dependencies between data center components and the policies governing management and construction of those environments (OASIS 2004). As an application of the XML, it provides a platform-independent specification, and is not restricted to any product but to the context of a data center. As an example of an approach that tries to merge these ideas on an abstract system level, SysML focuses on the specification, analysis, design, verification and validation of systems and systems-of-systems based on UML (SysML Partners 2005). Trying to focus on all kinds of systems in general, this approach has a big potential, but does take neither security nor federated identities into account.

Approaches that specifically target the systematic modeling and operation of Web-based systems are covered by the discipline Web Engineering. Many of them provide design methologies for dealing with the various aspects of Web applications, including navigation, interaction and business processes. For example, WebML, OOHDM, UWE and HERA (Kappel, Pröll et al. 2006) stress the hypermedia aspect of Web applications, focusing an application's composition from individual pages and navigatable nodes rather than a Web-based system's composition from individual services and applications. WebSA (Meliá and Cachero 2004) applies the model-driven development paradigm by combining architectural models with the design methods mentioned above. Although WebSA does not suffer from the model to system and system to model problem, due to the model-driven approach, there is no integration of security and federated identities as a requirement.

\section{WAM MODELING APPROACH}

In the following sub sections, we describe the overall WAM modeling framework, give an overview of the graphical notation of WAM and briefly describe an approach to enrich system descriptions with necessary technical details.

\subsection{Modeling Complex Systems}

With reference to the desired reduction of complexity in system engineering, it is not advisable to try covering all aspects of a federation's architecture in one model. Therefore, our approach takes the separation of system concerns into account and is based on a framework of multiple models that each target different concerns or system layers (cf. Figure 1). In this context, WAM is understood as the foundation model that is intended to cover the most vital aspects of federated systems. More layers can be added as extensions, possibly by third-parties, to describe concepts that are not part of WAM and that might be specific to certain tools for processing the contained information. The various models are not totally independent, as the modeling entities residing in different layers can often be related to each other with inter-model relationships.

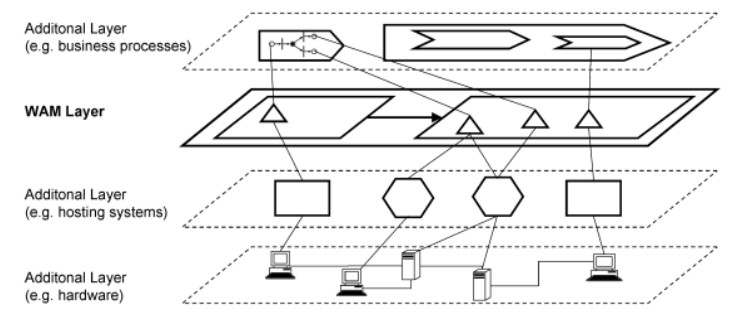

Figure 1: Model layers with inter-model relationships

The framework restricts the potential model structure by prescribing as a minimal condition the taxonomy of model constructs: All Model elements, (usually represented by dedicated symbols) are either Entities, which can be mapped to objects or concepts of the modeled world, or directed Relationships, which link multiple entities, possibly across different model layers. As a fixed built-in relationship, entities can be assigned to parent entities of which they are a part of. This introduces a 
structural hierarchy among entities to account for the architectural focus of the models.

\subsection{WAM Graphical Notation}

In order to account for the integration of security concerns into architecture models, WAM adopts general concepts from the state of the art of federated identity and access management protocols. Currently, there are several specifications being worked on that follow an approach based on socalled security tokens, including WS-Federation (Bajaj, Della-Libera et al. 2003), SAML (Maler, Mishra et al. 2003) and the Liberty Alliance project (Liberty Alliance Group 2004). Common to all specifications, such tokens take generally the form of digitally signed XML documents that contain security-relevant statements which can be exchanged between different system entities. The statements are usually either a proof of identity (e.g. of a user that wants to $\log$ into the system) or a proof of privileges (like e.g. the set of roles belonging to a user). As such, the token provides a basis for access control decisions for the protection of Web services and Web applications. As a major advantage, this allows for authentication and authorization tasks to be distributed and delegated to individual system parts as needed. WAM abstracts from the concrete flow of security tokens, in order to hide unwanted complexity and concentrate on the most important aspects of the modeled federations.

Rather then applying universal modeling techniques, as e.g. defining UML stereotypes, we took a domain-specific approach to focus on representations that can be drawn in a simple pen and paper fashion. Figure 2 contains the symbols of the most important model elements. Further details about the model, can be found in (Meinecke, Gaedke et al. 2005; Meinecke, Gaedke et al. 2006)
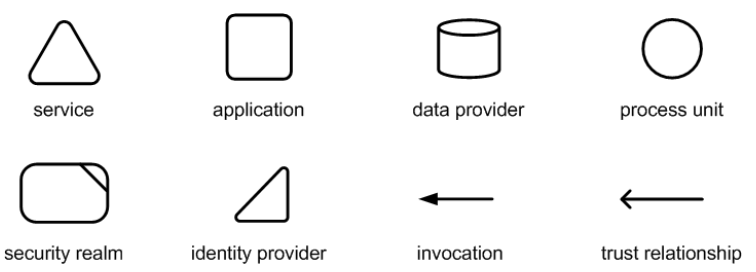

Figure 2: Symbols for important WAM modeling elements

The security realm represents organizational boundaries and with it the zones of control over the owned Web-based systems. It is equipped with exactly one designated security token service (STS). This service acts as the source for the tokens required to use the realm's resources and is as such the central authority for access control decisions. As a counterpart to the STS, the identity provider (IP) is a security token service specialized on authenticating anonymous requestors. Based on the authentication, the IP generates tokens that can then be presented at the STS for authorization requests. The services represent the distributed components that are provided by the different involved federation partners, usually in the form of SOAP Web services that expose their functionality through a defined WSDL interface. From the user point of view, the interaction with the overall system takes place through the interfaces provided by (Web) applications. In addition to that, WAM also addresses important system parts that are not directly Web-capable. In cases where it is useful to distinguish between a service and the underlying component that serves as the actual data source, this can be modeled with a separate data provider, like e.g. databases and wrapped legacy systems. If a connected system performs functionality beyond data management, then it is represented with a process unit symbol, like e.g. for an output management solution that passes on messages via mail or fax. Potential accesses on services and applications are stated with invocation links. In order to form federations, trust relationships can be established between separate realms. Semantically, this extends the area of validity of the trusted realm's security tokens to the trusting realm. In this context, the STS acts as a gateway that accepts foreign tokens and maps them to locally valid claims, based on a set of pre-defined rules.

As an example, Figure 3 contains the design of two federated university Web portals for students and library users. Both portals provide their content with the help of Web services, with Stud Portal also integrating the functionality of a service from the other realm. This is enabled by the trust relationship running in the opposite direction. As an additional form of cooperation, this setup also allows students that have already logged in at realm $A D M$ to perform tasks at the portal in realm $U B$ without any additional authentication steps (single sign on).

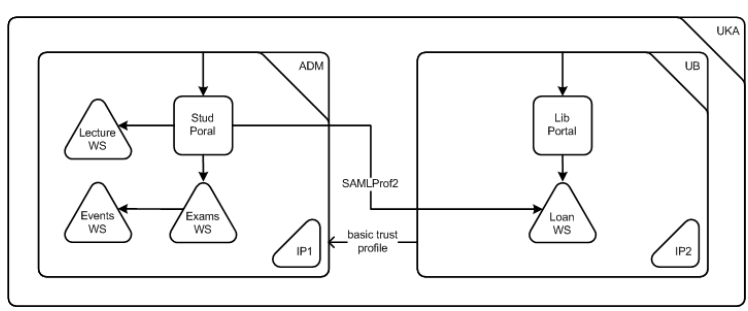

Figure 3: WAM example scenario 


\subsection{Specification of technical details}

As the application of the model to real-world scenarios has shown, it often becomes necessary to include characteristics of the system that are difficult to express in visual-only notations. For example, the Web service protocols cannot always be referred to by a simple label like "SOAP over HTTP", as there exist a huge number of options concerning e.g. cryptographic operations or ways of requesting and passing on security tokens. A similar need for annotation exists with respect to other modeling elements, as e.g. trust relationships. We suggest including such details in reusable profiles outside the graphical notation, which are referred to from within the diagrams by labels. As a first approach to profile specification, we have applied the Object Constraint Language (OCL) (Warmer and Kleppe 1999) in correspondence to the way OCL supplements UML diagrams. For example, referring to the invocation label in Figure 3, an OCL constraint with securityspecific requirements to the service invocation can make fine-grained statements about the signatures, encrypted information and tokens included in the SOAP message:

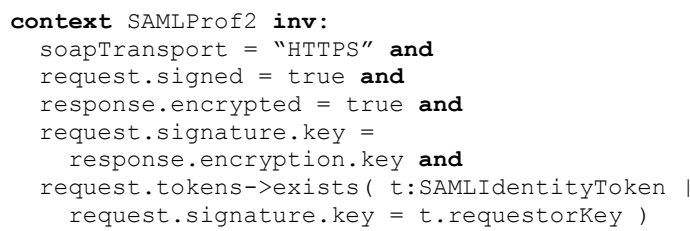

The formal basis for the OCL restrictions is established by a metamodel providing the properties of the modeling elements that can be put together to form expressions (Meinecke, Gaedke et al. 2005).

\section{WAM-XML LANGUAGE}

The graphical notation of WAM focuses on diagrams that are relatively easy to draw and comprehend. As a means for documenting the system's architecture, they form the basis for communicating the models between stakeholders, e.g. during the design process. Later at operation time, the changes that inevitably affect the evolving system cause the mentioned gap between the modeled world and the model. While OCL expressions help humans to add system details that are also relevant for the implementation at runtime (e.g. protocol restrictions), their complex syntax render them inappropriate for further machine-based processing, automation and code generation. Instead, we designed a language that is capable of describing the modeling concepts from the previous section, and at the same time forms the basis for tools and support systems. The language (called WAM-XML in the following) is implemented as an application of the XML, and as such profits from its manifold capabilities, especially in the context of large-scale heterogeneous systems. WAM-XML addresses both the standard WAM elements and supports multiple modeling layers. Hence, a corresponding XML document may contain model instances from different layers at the same time. Documents with models that are related to each other can also be aggregated to more comprehensive representations of the overall system.

The definition of the XML notation is given in the form on an XML schema, which incorporates existing XML-based specifications where appropriate to improve the overall interoperability and the applicability of standard tools. For example, WAM-XML comprises a metadata concept that is based on the Dublin Core Metadata Initiative (Andresen 2003), a de-facto standard defining a set of common meta-level attributes. Moreover, the realization of the WAM relationship concept makes use of the XLink specification (DeRose, Maler et al. 2000) that describes standardized ways of linking together resources in XML documents. To account for the various applied standards, as well as for the different parts of the WAM modeling framework, WAM-XML divides the defined XML attributes and elements into multiple namespaces. Thus, there is one namespace for the modeling framework (abbreviated with core in the following), a separate one for the actual WAM concepts (wam), as well as extra namespaces for the adopted standards Dublin Core $(d c)$ and XLink (xlink). In the case of custom model extensions, new namespaces can be introduced to clearly distinguish the additional modeling layers from the pre-defined standard parts. Consequently, tools that consume WAM-XML documents only need to understand document parts that correspond to a limited number of namespaces and ignore the others.

As specified by the WAM modeling framework, there is the common concept of the modeling element in all WAM-compliant models. In the schema, this is reflected by the core type Element, from which all other modeling types must inherit (1). As a result of this inheritance hierarchy, all parts of the model can be tagged with the Dublin Core properties defined by Element, allowing for uniform ways of processing different constructs. The required Identifier property for example contains a Uniform Resource Identifier (URI) that can be used 
to uniquely address a certain element. As a more readable form of representation, the Title coincides with the string that labels the symbols of most modeling elements (e.g. the name of a security realm).

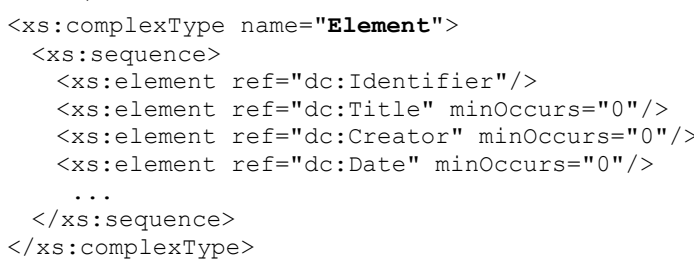

Representing the class of modeling elements that link together different entities, the Relationship type inherits from Element and adds further XLinkcompliant attributes (2). According to the XLink specification, the resources to be connected are not addressed directly, as e.g. by an identifier. Instead, the from and to attributes contain XLink labels that serve as placeholders for a separately declared group of resources. For this purpose, the WAM-XML schema includes the Selector type that can be used to map a label to a URI, or even to multiple URIs, if more than one selector is defined with the same label. Typically, this URI takes the form of an XPointer expression that describes the positions of a set of XML elements. In addition to the standard compliance, this approach has the advantage that the same relationship can have multiple origins and destinations (n:m relationships). For example, this allows for a concise statement of the fact that all realms in a model trust a dedicated central realm. The addressed resources may reside in different XML files, providing ways of distributing the model on multiple documents, possibly owned by different federation partners.

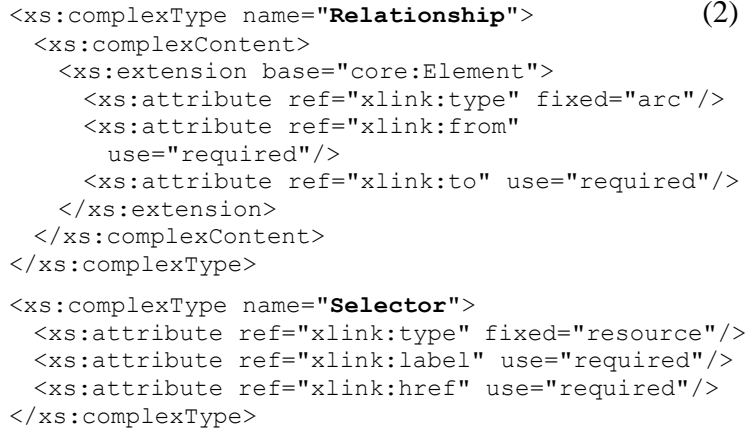

Derived from the Relationship type, the Invocation is an example of a concrete modeling element of the WAM namespace (3). The corresponding schema type declares properties that include the actual layerspecific model information, in addition to the metalevel information of the parent types. In this case, this includes the underlying transport level protocol applied to deliver the SOAP message, and SOAPContext elements to describe SOAP-specific restrictions to the request and response messages.

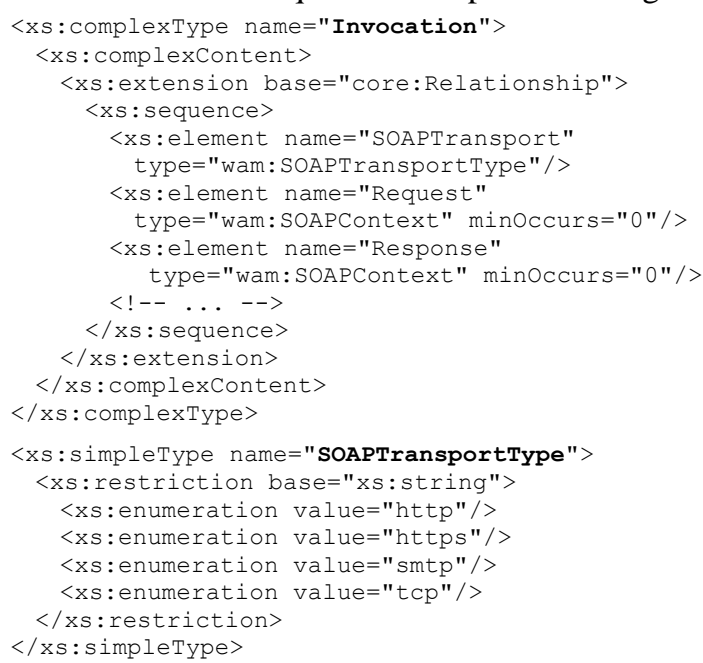

To demonstrate, how the schema types are instantiated in WAM-XML documents, we show an extract from an example model (4). The document contains elements both from the WAM layer (wam), as well as from the model extension $(h s t)$. In this particular scenario, there is a Web service WS1 that is hosted by a Web server labeled as Serverl. This is expressed with an inter-model relationship defined within the hosting layer namespace. As prescribed by the relationship concept, two labels LabelWS1 and LabelS1 are declared, which in this simple example can be directly mapped to one XMLelement each. The mapping is achieved with an XPointer expression that uses the identifier introduced by the Dublin Core metadata concept as a unique key for addressing. Although the document contains non-standard extensions, a tool developed without any knowledge about the hst namespace would still be able to process the rest of the document, simply by ignoring any unknown elements.

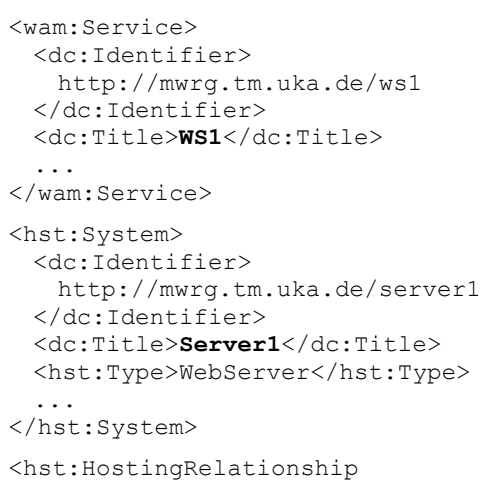




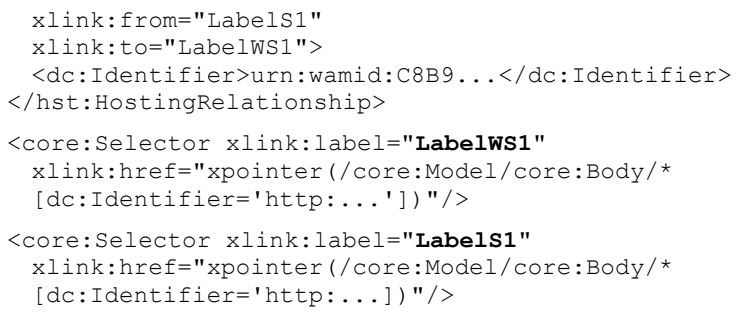

While the presented XML format is not necessarily intuitive to write down manually, it provides a solid basis for applications that work with the modeling information at the operation time of the federated system. With a similar expressiveness of both representations, models can be transformed from one into the other and vice versa.

\section{WAM SERVICE}

In the context of the overall goal to facilitate the design and evolution of Web-based federations, the existence of a machine-readable modeling language is only a first step. Additionally, the modeling data has to be exposed and made available within the distributed solution, calling for the presence of a supporting infrastructure. Our idea regarding this is to apply the same technology that is already in use for the functional parts of the architecture and provide a Web service for querying and changing the model. Similarly to a UDDI node, this infrastructure service takes the role of a central registry, at which the partners of the federation publish their components and relationships. Unlike UDDI, the entries are not related to the provided functionality, but instead to the federation- and access-control-specific aspects that is not covered by UDDI.

The outlined WAM Service has been applied within the service-oriented integration project KIM conducted at the University of Karlsruhe (University of Karlsruhe 2005). The scope of integration covers 11 faculties as well as cooperations with other universities in the context of the Bologna Process (European Union 2005), which supports the mobility of students, teachers and researchers within the European Union by introducing common quality standards. As one of a group of infrastructure Web services, the WAM service acts as a source of architectural information about the growing network of services provided by the different university departments. This group includes a UDDI-based service registry, a status log service providing the health history of monitored resources, as well as other third-party services. A common addressing concept among the data objects of the infrastructure services ensures that system information from different sources can be related to each other. For example, this allows for looking up an application described in WAM and afterwards querying the status $\log$ for health status information about that same application.

On top of the WAM infrastructure service, two applications have been implemented that work directly on the provided model information and have successfully been deployed in several projects. As a means for supporting model engineers in creating and modifying system descriptions, we customized Microsoft Visio with dedicated support for WAM diagrams. The drag \& drop interface editor (cf. Figure 4) allows the placement of the pre-defined model symbols and their annotation with additional attributes, according to the data model presented in the previous section. With the help of the XMLsupport already built into Visio, we added an XSLTbased transformation engine to generate WAMXML from the diagrams and write the resulting code into the model database managed by the WAM Service.

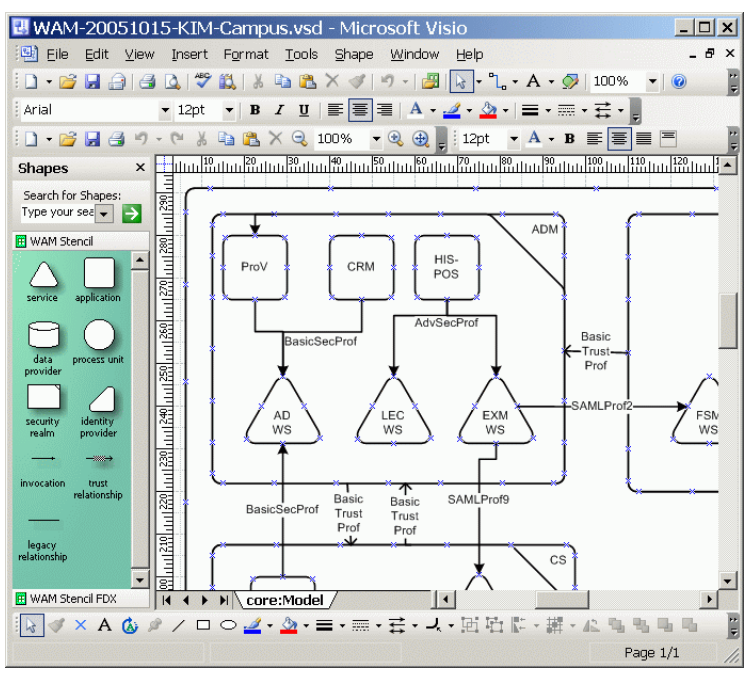

Figure 4: Diagram authoring support

While the model in the database is being updated manually with Visio or automatically through additional support tools, it becomes important to keep track of the ongoing changes within the federation. One way to do this in a standardized manner is to provide an RSS feed that serves as a means for publishing events - in other words: the federation is blogging about its existence. Thus, an overview is provided of new services joining the 
federation, changing trust relationships, the rerouting of service invocations etc.

We consider the two presented applications as a proof of concept for the approach to expose the model via a service that is itself a part of the federation it supports. The Visio-based diagram authoring tool and other related infrastructure components can be downloaded at http://mwrg.tm.uni-karlsruhe.de/downloadcenter/.

\section{CONCLUSION}

We presented WAM as an approach to model the architecture of federated Web-based applications with a special focus on token-based access control concepts. As key challenges to such a model, we identified the need for integrating security aspects into the model, for hiding unwanted complexity and for linking the model close to the modeled evolving system. The WebComposition Architecture Model, is founded on token-based access control concepts identified in current specifications. As a machinereadable representation of WAM, an XML format has been defined. This XML-based system information is exposed through an infrastructure Web service, on top of which tools can be built to support federated applications at operation time.

One possible extension of the described work would be the definition of a UML profile for WAM to enable the applicability of standard UML modeling tools. As mentioned, the machine-readable model and the developed support service offer potential for applications that go beyond the two demonstrated tools. Therefore, in the future we will add systems that provide a higher degree of automation and code generation, like e.g. producing configuration files for the participating services and applications directly from the model.

\section{REFERENCES}

Andresen, L., 2003. Dublin Core Metadata Element Set, Version 1.1: Reference Description, Dublin Core Metadata Initiative (DCMI).

Bajaj, S., G. Della-Libera, et al., 2003. Web Services Federation Language (WS-Federation). http://www106.ibm.com/developerworks/webservices/library/wsfed/ (14.10.2004).

Bass, L., P. Clements, et al., 1998. Software Architectures in Practice. Reading, USA, Addison-Wesley.
Cameron, K., 2005. The Laws of Identity. http://msdn.microsoft.com/library/enus/dnwebsrv/html/lawsofidentity.asp (29.10.2005).

DeRose, S., E. Maler, et al., 2000. XML Linking Language (XLink) Version 1.0, World Wide Web Consortium.

European Union, 2005. The Bologna Process. http://europa.eu.int/comm/education/policies/educ/bol ogna/bologna_en.html (23.02.2006).

Gootzit, D. and G. Phifer, 2003. Gen-4 Portal Functionality: From Unification to Federation. Stamford, CT.

Hermanns, J., C. Jänsch, et al., 1999. "Architekturmanagement im Großunternehmen." OBJEKTspektrum(4/99).

Kappel, G., B. Pröll, et al., 2006. Web Engineering: The Discipline of Systematic Development, Wiley.

Kirchner, L., 2005. Cost Oriented Modelling of ITLandscapes: Generic Language Concepts of a Domain Specific Language. Workshop on Enterprise Modelling and Information Systems Architectures (EMISA '05), Klagenfurt, Austria.

Liberty Alliance Group, 2004. Liberty Alliance Specifications.

http://www.projectliberty.org/resources/specifications. php (18.10.2004).

Maler, E., P. Mishra, et al., 2003. Assertions and Protocol for the OASIS Security Assertion Markup Language (SAML) V1.1. http://www.oasis-open.org/specs/ (18.10.2004).

Meinecke, J., M. Gaedke, et al., 2006. Capturing the Essentials of Federated Systems. 15th International World Wide Web Conference ( $W W W$ ), Edinburgh, UK, ACM.

Meinecke, J., M. Gaedke, et al., 2005. A Web Engineering Approach to Model the Architecture of InterOrganizational Applications. Conference on Component-Oriented Enterprise Applications (COEA 2005), Erfurt, Germany, Gesellschaft für Informatik.

Meliá, S. and C. Cachero, 2004. An MDA Approach for the Development of Web Applications. 4th International Conference of Web Engineering (ICWE 2004), Munich, Germany.

Microsoft, 2003. Dynamic Systems Initiative Roadmap. http://www.microsoft.com/dsi (14.10.2004).

OASIS, 2004. Data Center Markup Language Framework Specification. http://www.dcml.org/technical_info/ (03.11.2004).

Park, J. and S. Ram, 2004. "Information Systems Interoperability: What Lies Beneath?" $A C M$ Transactions on Information Systems (TOIS) 22(4): 595-632.

SysML Partners, 2005. Systems Modeling Language (SysML) Specification Version 0.9 Draft. http://www.sysml.org/ (17.10.2005).

University of Karlsruhe, 2005. KIM Project Homepage. http://www.kim.uni-karlsruhe.de/ (24.04.2005).

Warmer, J. and A. Kleppe, 1999. The Object Constraint Language, precise modeling with UML. Reading, Mass., Addison-Wesley Pub Co. 